\title{
ON THEOREMS OF BRAUER-NESBITT AND BRANDT FOR CHARACTERIZATIONS OF SMALL BLOCK ALGEBRAS
}

\author{
SHIGEO KOSHITANI AND TARO SAKURAI
}

\begin{abstract}
In 1941, Brauer-Nesbitt established a characterization of a block with trivial defect group as a block $B$ with $k(B)=1$ where $k(B)$ is the number of irreducible ordinary characters of $B$. In 1982, Brandt established a characterization of a block with defect group of order two as a block $B$ with $k(B)=2$. These correspond to the cases when the block is Morita equivalent to the one-dimensional algebra and to the non-semisimple two-dimensional algebra, respectively.

In this paper, we redefine $k(A)$ to be the codimension of the commutator subspace $K(A)$ of a finite-dimensional algebra $A$ and prove analogous statements for arbitrary (not necessarily symmetric) finite-dimensional algebras. This is achieved by extending the Okuyama refinement of the Brandt result to this setting. To this end, we study the codimension of the sum of the commutator subspace $K(A)$ and $n$th Jacobson radical $\operatorname{Rad}^{n}(A)$. We prove that this is Morita invariant and give an upper bound for the codimension as well.
\end{abstract}

\section{CONTEnTS}

1. Introduction

2. Morita invariance

3. Upper bound

4. Small algebras

Appendix A. The Chlebowitz theorem

Acknowledgements

References

\section{INTRODUCTION}

Modular representation theory of finite groups aims to understand the structure of a block of a finite group algebra and its invariants. The complexity of the representations of a block $B$ is measured by invariants including the defect group $P$, the number of irreducible ordinary characters $k(B)$, the number of irreducible modular characters $\ell(B)$, and the Cartan matrix $C_{B}$ of the block $B$. A semisimple block, a block of the simplest kind, is characterized as a block of defect zero; The

Date: November 9, 2018.

2010 Mathematics Subject Classification. $16 \mathrm{G} 10$ (16P10, 16E40, 20C20).

Key words and phrases. codimension, commutator subspace, finite-dimensional algebra, Morita invariant, Morita equivalence.

The first author was partially supported by the Japan Society for Promotion of Science (JSPS), Grant-in-Aid for Scientific Research (C)15K04776, 2015-2018. 
characterization has the following equivalent condition, which was essentially due to Brauer-Nesbitt [4, Section 11].

$$
P \cong 1 \Longleftrightarrow k(B)=1 .
$$

A few decades later Brandt [2, Theorem $\mathrm{A}]$ proved similar.

$$
P \cong \mathbb{Z} / 2 \mathbb{Z} \Longleftrightarrow k(B)=2(\text { and } \ell(B)=1) \text {. }
$$

This condition characterizes a block that is Morita equivalent to the group algebra of $\mathbb{Z} / 2 \mathbb{Z}$. For textbook accounts for these, see the Landrock textbook [11, Section I.16]. We call a block $B$ small if $k(B)$ is small.

The aim of this paper is to gain a better understanding of the above results by generalizing to arbitrary finite-dimensional algebras. Throughout this paper, $A$ denotes a finite-dimensional algebra over a field $F$ and $\left\{S_{i} \mid 1 \leq i \leq \ell(A)\right\}$ a complete set of pairwise non-isomorphic simple right $A$-modules. An obstacle for generalization is that there is no established notion of $k(A)$. To overcome this difficulty we introduce the following definition.

Definition 1.1. Let $K(A)$ be the commutator subspace of $A$ (i.e., the $F$-subspace of $A$ spanned by $x y-y x$ for all $x, y \in A$ ). Then we define

$$
k(A):=\operatorname{codim} K(A) .
$$

The new definition of $k(A)$ for an algebra $A$ coincides with the old $k(B)$ for a block $B$ by Külshammer [8, Lemma A(ii)]. Hence $k(A)$ is supposed to measure the complexity of the representations of the algebra $A$. Indeed, we are able to prove that this is the case if $k(A)$ is small.

Theorem 1.2 (See also Theorem A.1). Suppose that $F$ is a splitting field for A. Then the following statements are equivalent.

(i) $A$ is Morita equivalent to $F$.

(ii) $k(A)=1$.

Theorem 1.3 (See also Theorem A.1). Suppose that $F$ is a splitting field for A. Then the following statements are equivalent.

(i) $A$ is Morita equivalent to $F[X] /\left(X^{2}\right)$.

(ii) $k(A)=2$ and $\ell(A)=1$.

Note that we cannot omit the condition $\ell(A)=1$ in the above (Note 4.4). If, among others, $A$ is non-semisimple symmetric then we can omit the condition (Proposition 4.5). We also remark that a natural candidate $k^{*}(A):=\operatorname{dim} Z(A)$, the dimension of the center, does not work here. Consider the $F$-algebra $T_{n}$ of lower triangular matrices of degree $n \in \mathbb{N}$. Then $k^{*}\left(T_{n}\right)=1$ and the analog is no longer true.

These theorems are obtained from a study of the codimension of the $F$-subspace of $A$ defined by

$$
K R^{n}(A):=K(A)+\operatorname{Rad}^{n}(A) \quad(n \in \mathbb{N})
$$

where $\operatorname{Rad}^{n}(A)$ denotes the $n$th Jacobson radical of $A$. The following has been known for finite-dimensional symmetric algebras, while we extend it for arbitrary finite-dimensional algebras.

Theorem 1.4 (See also Note 3.5). Suppose that $F$ is a splitting field for A. Then the following holds. 
(i) $\operatorname{codim} K R^{1}(A)=\ell(A)$.

(ii) $\operatorname{codim} K R^{2}(A)=\ell(A)+\sum_{1 \leq i \leq \ell(A)} \operatorname{dim} \operatorname{Ext}^{1}\left(S_{i}, S_{i}\right)$.

(iii) $\ell(A)+\sum_{1 \leq i \leq \ell(A)} \operatorname{dim} \operatorname{Ext}^{1}\left(S_{i}, S_{i}\right) \leq k(A) \leq \operatorname{tr} C_{A}$.

This paper is organized as follows. First, Section 2 is devoted to prove Morita invariance (Theorem 2.1). In Section 3, we establish an upper bound for the codimension of $K R^{n}(A)$ in Theorem 3.4 and prove Theorem 1.4. Finally, Section 4 provides a characterization of truncated polynomial algebras (Theorem 4.2) using Theorems 1.4 and 2.1. Then characterizations of small algebras (Theorems 1.2 and 1.3) follow immediately.

\section{Morita inVariance}

This section is devoted to prove Morita invariance of the codimension of $K R^{n}(A)$. See also Sakurai [14, Theorem 2.3] for its "dual".

Theorem 2.1. Let $A$ and $B$ be Morita equivalent finite-dimensional algebras over a field $F$. Then there is an $F$-linear isomorphism $A / K(A) \rightarrow B / K(B)$ inducing the $F$-linear isomorphism $A / K R^{n}(A) \rightarrow B / K R^{n}(B)$ for every $n \in \mathbb{N}$.

Proof. It suffices to prove the case for $A$ and its basic algebra $B:=e A e$ because Morita equivalent basic algebras are isomorphic. Since the basic idempotent $e \in A$ is full, there exist elements $u_{k}, v_{k} \in A$ such that

$$
1=\sum_{k} u_{k} e v_{k}
$$

Then define $F$-linear maps

$$
A / K(A) \underset{\sigma}{\stackrel{\tau}{\rightleftarrows}} B / K(B)
$$

by

$$
\begin{array}{llrl}
\tau(a+K(A)) & =\sum_{k} e v_{k} a u_{k} e+K(B) & & (a \in A) \\
\sigma(b+K(B)) & =b+K(A) & & (b \in B) .
\end{array}
$$

We claim that $\tau$ and $\sigma$ are well-defined and mutually inverse. Indeed, for $x, y \in A$ we have

$$
\begin{aligned}
\sum_{k} e v_{k}(x y-y x) u_{k} e & =\sum_{k} e v_{k} x y u_{k} e-\sum_{l} e v_{l} y x u_{l} e \\
& =\sum_{k, l} e v_{k} x u_{l} e v_{l} y u_{k} e-\sum_{l, k} e v_{l} y u_{k} e v_{k} x u_{l} e \\
& =\sum_{k, l}\left(\left(e v_{k} x u_{l} e\right)\left(e v_{l} y u_{k} e\right)-\left(e v_{l} y u_{k} e\right)\left(e v_{k} x u_{l} e\right)\right) \\
& \in K(B) .
\end{aligned}
$$

Hence $\tau$ is well-defined. It is routine to check the other parts. 
These linear isomorphisms induce linear maps $\tau_{n}$ and $\sigma_{n}$ commuting the following diagram where $\pi_{n,-}$ denotes the canonical epimorphism.

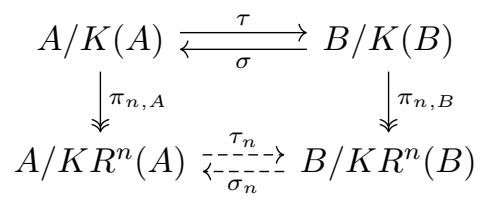

These maps are well-defined and diagram chase reveals that these maps are mutually inverse since $\pi_{n,-}$ is an epimorphism.

\section{UPPER BOUND}

In this section we establish an upper bound for the codimension of $K R^{n}(A)$, which extends the result of Otokita [13] (Theorem 3.4). As a corollary we prove Theorem 1.4.

Notation 3.1. For a basid 1 set of primitive idempotents $\left\{e_{i} \mid 1 \leq i \leq \ell(A)\right\}$ of $A$, set

$$
\operatorname{aCyc}(A):=\sum_{\substack{1 \leq i, j \leq \ell(A) \\ i \neq j}} e_{i} A e_{j} \quad \text { and } \quad \operatorname{Cyc}^{\geq n}(A):=\sum_{1 \leq i \leq \ell(A)} e_{i} \operatorname{Rad}^{n}(A) e_{i} .
$$

Lemma 3.2. If $A$ is basic then

$$
\sum_{1 \leq i \leq \ell(A)} \operatorname{dim} e_{i} A e_{i} / e_{i} \operatorname{Rad}^{n}(A) e_{i}=\operatorname{codim}\left(\operatorname{aCyc}(A)+\mathrm{Cyc}^{\geq n}(A)\right) .
$$

Proof.

$$
\begin{aligned}
\sum_{i} & \operatorname{dim} e_{i} A e_{i} / e_{i} \operatorname{Rad}^{n}(A) e_{i} \\
& =\operatorname{dim} \sum_{i} e_{i} A e_{i} / \sum_{i} e_{i} \operatorname{Rad}^{n}(A) e_{i} \\
& =\operatorname{dim} \sum_{i, j} e_{i} A e_{j} /\left(\sum_{i \neq j} e_{i} A e_{j}+\sum_{i} e_{i} \operatorname{Rad}^{n}(A) e_{i}\right) \\
& =\operatorname{codim}\left(\operatorname{aCyc}(A)+\mathrm{Cyc}^{\geq n}(A)\right) .
\end{aligned}
$$

Proposition 3.3. If $A$ is basic then the following statements are equivalent.

(i) $\operatorname{codim} K R^{n}(A)=\sum_{1 \leq i \leq \ell(A)} \operatorname{dim} e_{i} A e_{i} / e_{i} \operatorname{Rad}^{n}(A) e_{i}$.

(ii) $K(A) \subseteq \operatorname{aCyc}(A)+\mathrm{Cyc}^{\geq n}(A)$.

Proof. First, note that

$$
K R^{n}(A) \supseteq \operatorname{aCyc}(A)+\mathrm{Cyc}^{\geq n}(A) .
$$

(i) $\Longrightarrow$ (ii) By (3.1) and Lemma 3.2, we have $K(A) \subseteq K R^{n}(A)=\operatorname{aCyc}(A)+$ $\mathrm{Cyc}^{\geq n}(A)$.

\footnotetext{
${ }^{1}$ See 1 p. 305].
} 
(ii) $\Longrightarrow$ (i) By the hypothesis, we have the following.

$$
\begin{aligned}
K R^{n}(A) & \subseteq \operatorname{aCyc}(A)+\operatorname{Cyc}^{\geq n}(A)+\operatorname{Rad}^{n}(A) \\
& =\operatorname{aCyc}(A)+\sum_{i \neq j} e_{i} \operatorname{Rad}^{n}(A) e_{j}+\operatorname{Cyc}^{\geq n}(A)+\sum_{i} e_{i} \operatorname{Rad}^{n}(A) e_{i} \\
& =\operatorname{aCyc}(A)+\operatorname{Cyc}^{\geq n}(A) .
\end{aligned}
$$

Then $K R^{n}(A)=\operatorname{aCyc}(A)+\operatorname{Cyc}^{\geq n}(A)$ by (3.1). Thus, by Lemma 3.2 we have

$$
\begin{aligned}
\operatorname{codim} K R^{n}(A) & =\operatorname{codim}\left(\operatorname{aCyc}(A)+\operatorname{Cyc}^{\geq n}(A)\right) \\
& =\sum_{i} \operatorname{dim} e_{i} A e_{i} / e_{i} \operatorname{Rad}^{n}(A) e_{i} .
\end{aligned}
$$

Theorem 3.4 (See Otokita 13 for blocks). Let $\left\{e_{i} \mid 1 \leq i \leq \ell(A)\right\}$ be a basic set of primitive idempotents of $A$. Then the following holds for every $n \in \mathbb{N}$.

$$
\operatorname{codim} K R^{n}(A) \leq \sum_{1 \leq i \leq \ell(A)} \operatorname{dim} e_{i} A e_{i} / e_{i} \operatorname{Rad}^{n}(A) e_{i}
$$

Furthermore, if the equality holds for some $n$ then so does for every $1 \leq m \leq n$; if the equality does not hold for some $n$ then so does not for every $m \geq n$.

Proof. By Theorem 2.1, we can assume $A$ is basic. We claim the map

$$
\begin{aligned}
\pi: \bigoplus_{1 \leq i \leq \ell(A)} e_{i} A e_{i} / e_{i} \operatorname{Rad}^{n}(A) e_{i} & \rightarrow A / K R^{n}(A), \\
\sum_{i}\left(a_{i}+e_{i} \operatorname{Rad}^{n}(A) e_{i}\right) & \mapsto \sum_{i} a_{i}+K R^{n}(A)
\end{aligned}
$$

is surjective. Let $a+K R^{n}(A) \in A / K R^{n}(A)$. Then we have the following.

$$
\begin{aligned}
a+K R^{n}(A) & =\sum_{i} e_{i} a e_{i}+K R^{n}(A)+\sum_{i \neq j} e_{i} a e_{j}+K R^{n}(A) \\
& =\sum_{i} e_{i} a e_{i}+K R^{n}(A)+\sum_{i \neq j}\left(e_{i} a e_{j}-e_{j} e_{i} a\right)+K R^{n}(A) \\
& =\sum_{i} e_{i} a e_{i}+K R^{n}(A) \\
& =\pi\left(\sum_{i} e_{i} a e_{i}+e_{i} \operatorname{Rad}^{n}(A) e_{i}\right) .
\end{aligned}
$$

Hence the proof of the inequality completes.

The latter statements follow from Proposition 3.3.

Proof of Theorem 1.4. Since the last statement (iii) is clear from (ii) and Theorem 3.4 we prove (i) and (ii) in the following. 
We first claim that equality holds in Theorem 3.4 for $n \leq 2$. By Theorem 2.1 we may assume that $A$ is basic. Then we have

$$
\begin{aligned}
K(A) & =\sum_{i, j, s, t}\left[e_{i} A e_{j}, e_{s} A e_{t}\right] \\
& \subseteq \operatorname{aCyc}(A)+\sum_{i, j}\left[e_{i} A e_{j}, e_{j} A e_{i}\right] \\
& =\operatorname{aCyc}(A)+\sum_{i \neq j}\left[e_{i} A e_{j}, e_{j} A e_{i}\right]+\sum_{i} K\left(e_{i} A e_{i}\right) \\
& \subseteq \operatorname{aCyc}(A)+\operatorname{Cyc}^{\geq 2}(A)+\sum_{i} K\left(e_{i} A e_{i}\right) \\
& \subseteq \operatorname{aCyc}(A)+\operatorname{Cyc}^{\geq 2}(A)+\sum_{i} \operatorname{Rad}^{2}\left(e_{i} A e_{i}\right) \\
& \subseteq \operatorname{aCyc}(A)+\operatorname{Cyc}^{\geq 2}(A) .
\end{aligned}
$$

Now the claim follows from Proposition 3.3 . Then we have the following.

$$
\begin{aligned}
\operatorname{codim} K R^{1}(A)= & \sum_{i} \operatorname{dim} e_{i} A e_{i} / e_{i} \operatorname{Rad}(A) e_{i} \\
= & \ell(A) ; \\
\operatorname{codim} K R^{2}(A)= & \sum_{i} \operatorname{dim} e_{i} A e_{i} / e_{i} \operatorname{Rad}^{2}(A) e_{i} \\
= & \sum_{i} \operatorname{dim} e_{i} A e_{i} / e_{i} \operatorname{Rad}(A) e_{i} \\
& \quad+\sum_{i} \operatorname{dim} e_{i} \operatorname{Rad}(A) e_{i} / e_{i} \operatorname{Rad}^{2}(A) e_{i} \\
= & \ell(A)+\sum_{i} \operatorname{dim} \operatorname{Ext}^{1}\left(S_{i}, S_{i}\right) .
\end{aligned}
$$

Notes 3.5. (i) If $F$ is an algebraically closed field of positive characteristic $p$, a well-known theorem of Brauer [3, (3A)] states

$$
\ell(A)=\operatorname{codim} T(A)
$$

where

$$
T(A):=\left\{a \in A \mid a^{p^{n}} \in K(A) \text { for some } n \in \mathbb{N}\right\} .
$$

Since $T(A)=K R^{1}(A)$ by Külshammer [8, Lemma B], Theorem[1.4](i) follows in this case. In fact, the part (i) is certainly not our contribution although is is sometimes stated only for positive characteristic case; It can be found, among others, in [11, Proposition I.13.3(i)].

(ii) Okuyama [12] proves essentially the same statement of Theorem 1.4](ii) for a block of a finite group algebra over an algebraically closed field of positive characteristic. (See also Koshitani [7, which is written in English.)

(iii) The inequality $\ell(A)+\sum_{1 \leq i \leq \ell(A)} \operatorname{dim} \operatorname{Ext}^{1}\left(S_{i}, S_{i}\right) \leq k(A)$ is an extension of Brandt [2, Theorem B]. (For a symmetric algebra, it can be proved that the equality holds if and only if its Loewy length is at most two. See also 14, Lemma 2.1].) For a block $B$ of a finite group algebra, the inequality $k(B) \leq \operatorname{tr} C_{B}$ is a direct consequence of $C_{B}={ }^{t} D_{B} \cdot D_{B}$ where $D_{B}$ is the decomposition matrix of $B$. See also Külshammer-Wada [10] for refinements in this case. 
Corollary 3.6. For a radical square zero algebra $A$ over a splitting field, we have $k(A)=\operatorname{tr} C_{A}$.

Proof. It suffices to prove $k(A) \geq \operatorname{tr} C_{A}$ by Theorem 1.4](iii)

$$
\begin{aligned}
& k(A) \geq \ell(A)+\sum_{i} \operatorname{dim} \operatorname{Ext}^{1}\left(S_{i}, S_{i}\right) \quad \text { (By Theorem 1.4 (iii) } \\
& =\ell(A)+\sum_{i} \operatorname{dim} e_{i} \operatorname{Rad}(A) e_{i} \quad\left(\operatorname{By}_{\operatorname{Rad}^{2}}(A)=0\right) \\
& =\sum_{i}\left(1+\operatorname{dim} e_{i} \operatorname{Rad}(A) e_{i}\right) \\
& =\sum_{i} \operatorname{dim} e_{i} A e_{i}=\operatorname{tr} C_{A} .
\end{aligned}
$$

\section{SMALl ALGEBRAS}

This section provides a characterization of truncated polynomial algebras $F[X] /\left(X^{n}\right)$ using Theorems 1.4 and 2.1. Then characterizations of small algebras (Theorems 1.2 and 1.3) follow immediately.

Lemma 4.1. Let $n \in \mathbb{N}$. Then the following statements are equivalent.

(i) $A$ is isomorphic to $F[X] /\left(X^{n}\right)$.

(ii) $A$ is n-dimensional basic local Nakayama algebra.

Theorem 4.2. Let $n \in \mathbb{N}$ and suppose that $F$ is a splitting field for $A$. Then the following statements are equivalent.

(i) $A$ is Morita equivalent to $F[X] /\left(X^{n}\right)$.

(ii) $k(A)=n$, $\operatorname{codim} K R^{2}(A) \leq 2$, and $\ell(A)=1$.

Proof. (i) $\Longrightarrow$ (ii) Clear by Theorem 2.1.

(ii) $\Longrightarrow$ (i) We may assume $A$ is basic. Since $\ell(A)=1$, we have the unique simple right $A$-module $S:=A / \operatorname{Rad}(A)$. By Theorem 1.4 ,

$$
\begin{aligned}
\operatorname{dim} \operatorname{Rad}(A) / \operatorname{Rad}^{2}(A) & =\operatorname{dim} \operatorname{Ext}^{1}(S, S) \\
& =\ell(A)+\operatorname{dim} \operatorname{Ext}^{1}(S, S)-\ell(A) \\
& =\operatorname{codim} K R^{2}(A)-\operatorname{codim} K R^{1}(A) \\
& \leq 2-1=1 .
\end{aligned}
$$

Thus $A$ is a basic local Nakayama algebra. By Lemma 4.1, we have $A \cong F[X] /\left(X^{m}\right)$ for some $m \in \mathbb{N}$. Since $A$ is commutative,

$$
n=\operatorname{codim} K(A)=\operatorname{dim} A=\operatorname{dim} F[X] /\left(X^{m}\right)=m .
$$

Therefore $A \cong F[X] /\left(X^{n}\right)$.

Proof of Theorem 1.2, (i) $\Longrightarrow$ (ii) Clear from Theorem 2.1.

(ii) $\Longrightarrow$ (i): Clear from Theorem 4.2 .

Proof of Theorem 1.3, (i) $\Longrightarrow$ (ii) Clear from Theorem 2.1

(ii) $\Longrightarrow$ (i) . Clear from Theorem 4.2 . 
Notes 4.3. Consider the four-dimensional basic local Frobenius algebra defined by

$$
A_{q}:=F\langle X, Y\rangle /\left(X^{2}, Y^{2}, X Y-q Y X\right) \quad(q \in F \backslash\{0,1\}) .
$$

Since $A_{q}$ is non-commutative, we have $k\left(A_{q}\right)=3$ by Theorems 1.2 and 1.3 . Hence, we may have infinitely many non-equivalent finite-dimensional algebras $A$ with $k(A)=3$ and $\ell(A)=1$ unlike the case $k(A)=1,2$. (This is because $A_{q} \cong A_{r}$ if and only if $\left\{q^{ \pm 1}\right\}=\left\{r^{ \pm 1}\right\}$ for $q, r \in F \backslash\{0\}$. See [15, p. 865].)

Notes 4.4. Consider the $n$-Kronecker algebra - the path algebra $F Q_{n}$ of the $n$-Kronecker quiver $Q_{n}$ defined by

$$
\circ \frac{\alpha_{1}}{\vdots} \underbrace{\frac{\alpha_{0}}{4}}_{\alpha_{n}} \circ
$$

Then $k\left(F Q_{n}\right)=\ell\left(F Q_{n}\right)=2$. Hence, we have infinitely many non-equivalent finite-dimensional algebras $A$ with $k(A)=\ell(A)=2$.

We have determined small algebras in Theorems 1.2 and 1.3 The other extreme case is that the algebra $A$ with $k(A)=\ell(A)$. These algebras are close to be semisimple to some extent.

Proposition 4.5. Suppose that $F$ is a splitting field for $A$. Then the following statements are equivalent.

(i) $k(A)=\ell(A)$

(ii) $\operatorname{Rad}(A) \subseteq K(A)$

In particular, $k(A)=\ell(A)$ holds if $A$ is semisimple. The converse also holds if $A$ is symmetric, commutative, or local.

Proof. (i) $\Longleftrightarrow$ (ii) Evidently $k(A)=\ell(A)$ if and only if $K(A)=K R^{1}(A)$, i.e., $\operatorname{Rad}(A) \subseteq K(A)$.

Symmetric case: Assume $A$ is symmetric with a symmetrizing form $\tau: A \rightarrow F$ and $k(A)=\ell(A)$. Then $\operatorname{Rad}(A) \subseteq K(A) \subseteq \operatorname{ker} \tau$. Hence we have $\operatorname{Rad}(A)=0$.

Commutative case: Assume $A$ is commutative and $k(A)=\ell(A)$. Then $\operatorname{Rad}(A) \subseteq$ $K(A)=0$.

Local case: Assume $A$ is local and $k(A)=\ell(A)$. Then $k(A)=\ell(A)=1$ and $A$ is Morita equivalent to $F$ by Theorem 1.2. Hence we have $\operatorname{Rad}(A)=0$.

\section{Appendix A. The Chlebowitz theorem}

Chlebowitz already studied similar problems and she proved the following stronger facts, which are essentially extensions of the previous works $[9,6$, to non-symmetric cases.

Theorem A.1 (Chlebowitz [5). Let $A$ be a finite-dimensional local algebra over an algebraically closed field. Then the following holds.

(i) If $k(A) \leq 3$ then $\operatorname{dim} A \leq 4$.

(ii) If $k(A)=4$ then $\operatorname{dim} A \leq 10$.

(iii) If $k(A)=5$ and $\operatorname{dim} \operatorname{Rad}(A) / \operatorname{Rad}^{2}(A) \leq 2$ then $\operatorname{dim} A \leq 12$. 


\section{ACKNOWLEDGEMENTS}

We wish to thank Yoshihiro Otokita for helpful discussions. This is part of the $\mathrm{PhD}$ thesis of the second author.

After the first version of this paper appeared on the arXiv, Burkhard Külshammer informed us that Theorems 1.2 and 1.3 in this version already appear in the unpublished PhD thesis by Marlene Chlebowitz [5]. We thank Burkhard Külshammer very much.

\section{REFERENCES}

[1] F. W. Anderson and K. W. Fuller, Rings and Categories of Modules, 2nd edn (SpringerVerlag, New York, 1992). doi 10.1007/978-1-4612-4418-9, MR 1245487, Zbl 0765.16001.

[2] J. Brandt, A lower bound for the number of irreducible characters in a block, J. Algebra $\mathbf{7 4}$ (1982) 509-515. doi 10.1016/0021-8693(82)90036-9, MR 647251 Zbl 0478.20009.

[3] R. Brauer, Zur Darstellungstheorie der Gruppen endlicher Ordnung (German), Math Z. 63 (1956) 406-444. doi 10.1007/BF01187950. MR 75953 Zbl 0093.03101.

[4] R. Brauer and C. Nesbitt, On the modular characters of groups, Ann. of Math. 42 (1941) 556-590. doi $10.2307 / 1968918$ MR 4042 Zbl 0027.15202

[5] M. Chlebowitz, Uber Abschätzungen von Algebreninvarianten (German), PhD Thesis, Universität Augsburg, 1991.

[6] M. Chlebowitz and B. Külshammer, Symmetric local algebras with 5-dimensional center, Trans. Amer. Math. Soc. 329 (1992) 715-731. doi 10.2307/2153960 MR 1025752 Zbl 0758.16004

[7] S. Koshitani, Endo-trivial modules for finite groups with dihedral Sylow 2-groups RIMS Kôkyûroku 2003 (2016) 128-132.

[8] B. Külshammer, Bemerkungen über die Gruppenalgebra als symmetrische Algebra (German), J. Algebra 72 (1981) 1-7. doi 10.1016/0021-8693(81)90308-2, MR 634613 Zbl 0472.16007

[9] B. Külshammer, Symmetric local algebras and small blocks of finite groups, J. Algebra $\mathbf{8 8}$ (1984) 190-195. doi 10.1016/0021-8693(84)90097-8. MR 741939 Zbl 0567.20007.

[10] B. Külshammer and T. Wada, Some inequalities between invariants of blocks, Arch. Math. 79 (2002) 81-86. doi 10.1007/s00013-002-8288-z, MR 1925373. Zbl 1011.20009

[11] P. Landrock, Finite Group Algebras and Their Modules, (Cambridge University Press, Cambridge, 1983). doi 10.1017/CBO9781107325524, MR 737910 Zbl 0523.20001.

[12] T. Okuyama, $\operatorname{Ext}^{1}(S, S)$ for a simple $k G$-module $S$ (Japanese), in Proceedings of the Symposium "Representations of Groups and Rings and Its Applications," ed. S. Endo (1981), pp. 238-249.

[13] Y. Otokita, On diagonal entries of Cartan matrices of $p$-blocks, preprint (2016) 4pp. arXiv $1605.07937 \mathrm{v} 2$

[14] T. Sakurai, Central elements of the Jennings basis and certain Morita invariants, preprint (2017) 11pp. arXiv 1701.03799v3

[15] K. Yamagata, Frobenius algebras, in Handbook of Algebra, vol. 1, ed. M. Hazewinkel (Elsevier, Amsterdam, 1996), pp. 841-887, doi 10.1016/S1570-7954(96)80028-3. MR 1421820, Zbl 0879.16008

Center for Frontier Science, Chiba University, 1-33 Yayoi-cho, Inage-Ku, Chiba, 2638522 , JAPAN.

E-mail address: koshitan@math.s.chiba-u.ac.jp

Department of Mathematics and Informatics, Graduate School of Science, Chiba University, 1-33, Yayoi-Cho, InAGe-Ku, Chiba-Shi, Chiba, 263-8522 JAPAN

E-mail address: tsakurai@math.s.chiba-u.ac.jp 